\title{
Effect of Blade Angle on Cavitation Phenomenon in Axial Pump
}

\section{Nabil H. Mostafa and Mohamed Adel ${ }^{\star}$}

Engineering Faculty, Mechanical Power Engineering Department, Zagazig University, Zagazig, 44519, Egypt

\begin{abstract}
The objective of this paper is to present 3-D numerical and experimental study of the effect of blade angle on the cavitation phenomenon. The numerical computation in cavitating flow carried out using the Navier-Stokes code (CFD-ACE+ 2008) is presented. The governing equations are discretized on a structured grid using an upwind difference scheme. The numerical simulation used the standard K- $\varepsilon$ turbulence model to account for the turbulence effect. Pressure distribution and vapor volume fraction were obtained numerically at variable blade angles. Also the performance curve of the axial pump was obtained at variable blade angles $60^{\circ}, 70^{\circ}$ and $80^{\circ}$. The numerical and experimental results showed that the cavitation phenomenon appeared at blade angle $60^{\circ}$ only. The computational code has been validated by comparing the predicted numerical results with the experimental ones. Besides, the predicted void growth and cavitation distribution on the impeller blade agreed with those visualized with high speed camera.
\end{abstract}

Keywords: Cavitation; Axial pump; CFD; High speed camera; Simulation; Blade angle

\section{Introduction}

The cavitation phenomenon is an important cause of wear and damages in turbo machines. Cavitation occurs during the flow of water in the presence of regions of high flow velocity when local static pressure decreases below the vapor pressure. Calculation of incipient cavitation is simple as one has just to find the lowest pressure indicating the cavitation inception once this lowest pressure reaches the vapor pressure. According to Sudsuansee et al. [1], cavitation may occur on the blade suction surface in region of low pressure or at the runner leading edge at off-design operation. In a limited number of studies the effort was to simulate the cavitation in axial pumps. Mostafa, et al. [2] used an energy model to predict the cavitation erosion in centrifugal pumps, where they predicted the hydrodynamic aspect of cavitation. Fukaya, et al. [3] predicted the cavitation performance of an axial pump, but the model used does not include a turbulence model. Sedlár et al. [4], performed analysis of cavitation phenomena in water and estimated its application on prediction of cavitation erosion in hydraulic machinery. In the same article, the experimental research of the cavitating flow aiming to the validation of the erosion potential model is also shortly described, as well as development of the nuclei-content measurement and the validation of the bubble nucleation model. De-sheng et al. [5] investigated numerically and experimentally the unsteady flow of

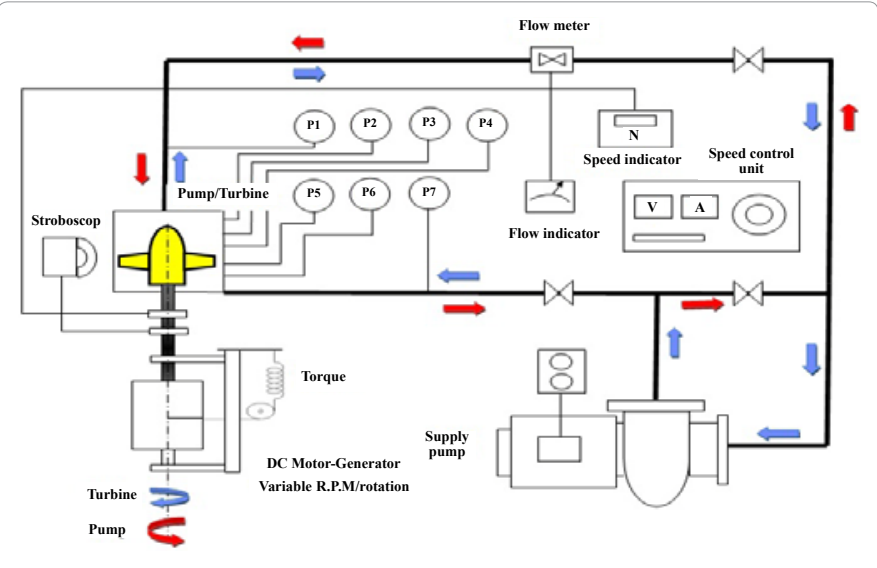

Figure 1: Schematic diagram of the experiment set-up. axial pump. The authors focused on the comparison between steady and unsteady results without being concerned with the cavitation phenomenon. Tabar and Poursharifi [6] performed pure experimental work to study the tip vortex cavitation inception in an axial flow pump, thus obtaining the pump characteristic curves through observation of the cavitation phenomenon with the aid of camera and stroboscope.

The main objective of this work is to study the effect of blade angle variations on the cavitation phenomenon in axial pump with specific interest in cavity geometry, pressure and void fraction fields. Besides, the effects of turbulence and fluid viscosity are included. The cavity shape over the blades and the 3-D flow field around the cavitating propeller will be determined. Finally, the pump performance curve will be obtained experimentally at variable blade angles.

\section{Experimental Setup}

In order to test the axial flow pump's performance under cavitation, a closed loop system including an axial flow turbo pump was used. A schematic diagram of the experimental setup is shown in figure 1.

The axial flow machine is provided with a comprehensive set of instruments (Table 1).

Pump's casing made out of plexi-glass enables for observation of fluid flow pattern inside the pump. Photos were taken with a high speed camera $1000 \mathrm{fps}$, showing the developed cavity along the blade leading edge for comparison purposes with the numerical results. The pump specifications are presented in table 2 .

\section{Theoretical Background}

The basic approach is to use standard viscous flow (Navier-Stokes)

*Corresponding author: Mohamed Adel, Engineering Faculty, Mechanical Power Engineering Department, Zagazig University, Zagazig, 44519, Egypt, E-mail: engm_adel@yahoo.com,nmostafa@zu.edu.eg

Received July 24, 2012; Accepted September 12, 2012; Published September 14,2012

Citation: Mostafa NH, Adel M (2012) Effect of Blade Angle on Cavitation Phenomenon in Axial Pump. J Appl Mech Eng 1:112. doi:10.4172/21689873.1000112

Copyright: (c) 2012 Mostafa NH, et al. This is an open-access article distributed under the terms of the Creative Commons Attribution License, which permits unrestricted use, distribution, and reproduction in any medium, provided the original author and source are credited. 
Citation: Mostafa NH, Adel M (2012) Effect of Blade Angle on Cavitation Phenomenon in Axial Pump. J Appl Mech Eng 1:112. doi:10.4172/21689873.1000112

\begin{tabular}{|l|c|c|c|}
\hline \multicolumn{1}{|c|}{ Device } & Variable & Unit & Uncertainty \\
\hline Pressure gauges & Pressure & bar & \pm 0.05 \\
\hline Flow meter & Flow rate & L/s & \pm 0.7088 \\
\hline Digital tachometer & rpm & Rpm & \pm 30.109 \\
\hline Voltmeter & Voltage & Volt & \pm 0.0005032 \\
\hline Ammeter & Current & Ampere & \pm 0.5001 \\
\hline Torque arm force & Force & N & \pm 1.063 \\
\hline
\end{tabular}

Table 1: Uncertainty for the used devices.

\begin{tabular}{|l|c|c|}
\hline \multicolumn{1}{|c|}{ Items } & unit & Value \\
\hline Tip diameter & $\mathrm{mm}$ & 101 \\
\hline Hub diameter & $\mathrm{mm}$ & 50 \\
\hline Hub to tip ratio & -- & 0.495 \\
\hline Number of blades & -- & 4 \\
\hline Blade angles & degree & $60-80$ \\
\hline Nominal speed & rpm & 3000 \\
\hline Motor power & $\mathrm{kW}$ & 2.2 \\
\hline
\end{tabular}

Table 2: Specifications of axial pump.

equations with provisions for variable density and a conventional turbulence model, such as $\mathrm{K}-\varepsilon$ model. The latter is a numerical model developed by CFD-ACE+ solving (Navier- Stokes) equations [7].

The mixture density $(\rho)$ is a function of vapor mass fraction $(f)$, which is computed by solving a transport equation simultaneously with the mass and momentum conservation equations. The $\rho-f$ relationship is:

$$
\frac{1}{\rho} \equiv \frac{f}{\rho_{v}}+\frac{1-f}{\rho_{1}}
$$

In two-phase flows, the use of vapor volume fraction ( $\alpha$ ) is also quite common. Therefore, it is deduced from $f$ as follows:

$$
\alpha=f \frac{\rho}{\rho_{v}}
$$

The transport equation for vapor is:

$$
\frac{\partial}{\partial t}(\rho f)+\nabla \bullet(\rho \vec{V} f)=\nabla \bullet(\Gamma \nabla f)+R_{e}-R_{c}
$$

The expressions of $R_{e}$ and $R_{c}$ have been derived from the reduced form of the Rayleigh-Plesset equation [8], which describes the dynamics of single bubble in an infinite liquid domain. The expressions for $R_{e}$ and $R_{c}$ are:

$$
\begin{aligned}
& R_{e}=C_{e} \frac{V_{h}}{\sigma} \rho_{l} \rho_{v} \sqrt{\frac{2}{3} \frac{p_{\text {sat }}-p}{\rho_{l}}}(1-f) \\
& R_{c}=C_{c} \frac{V_{h}}{\sigma} \rho_{l} \rho_{v} \sqrt{\frac{2}{3} \frac{p-p_{\text {sat }}}{\rho_{l}}} f
\end{aligned}
$$

Cavitation normally takes place in the vicinity of low pressure (or locally high velocity) regions, where turbulence effects are quite significant. In particular, turbulent pressure fluctuations have significant effect on cavitating flows. The magnitude of pressure fluctuations is estimated by using the following empirical correlation [9]:

$$
P_{\text {turb }}^{\prime}=0.39 \rho \mathrm{k}
$$

The phase-change threshold pressure value is:

$$
p_{v}=p_{\text {sat }}+0.5 p_{\text {turb }}^{\prime}
$$

It is well known that cavitating flows are sensitive to the presence of non-condensable gases. In most liquids, there is a small amount of non-condensable gases present in dissolved and/or mixed state. For example, laboratory water generally has $15 \mathrm{ppm}$ air dissolved in it. In other applications, e.g., marine propellers, etc., this amount may be considerably larger. In this model, the non-condensable gas is included by prescribing an estimated mass fraction at inlet. This value is held constant throughout the calculation domain. However, the corresponding density (and hence volume fraction) varies significantly with local pressure. The perfect gas law is used to account for the expansion (or compressibility) of gas; i.e.

$$
\rho_{\text {gas }}=\frac{W P}{R T}
$$

The calculation of mixture density (equation 1 ) is modified as:

$$
\frac{1}{\rho}=\frac{f_{v}}{\rho_{v}}+\frac{f_{g}}{\rho_{g}}+\frac{1-f_{v}-f_{g}}{\rho_{l}}
$$

Correspondingly, we have the following expression for the volume fractions of vapor $\left(\alpha_{\mathrm{v}}\right)$ and gas $\left(\alpha_{\mathrm{g}}\right)$ :

$$
\begin{aligned}
& \alpha_{v}=f_{v} \frac{\rho}{\rho_{v}} \\
& \alpha_{g}=f_{g} \frac{\rho}{\rho_{g}} \\
& \text { and, }
\end{aligned}
$$

$$
\alpha=1-\alpha_{v}-\alpha_{g}
$$

The combined volume fraction of vapor and gas (i.e., $\alpha_{v}+\alpha_{g}$ ) is referred as the Void Fraction ( $\alpha$ ). In practical applications, for qualitative assessment of the extent and location of cavitation, contour maps of void fraction $(\alpha)$ are important for bubbles location and shape determination.

\section{Computational Analysis}

Numerical simulation was performed using a 3-D Navier-Stokes code (CFD-ACE+, 2008) [10] to model the two phase flow field around a 3D impeller in a cavitating axial pump. This code [3] employs a homogenous flow approach, also known as Equal-Velocity-EqualTemperature (EVET) approach (CFD-ACE+, 2003).

The governing equations are discretized on a structured grid using an upwind difference scheme. The numerical simulation used the standard K- $\varepsilon$ turbulence model to account for the turbulence effect. The cavity shape was determined. Also, various variables were calculated, such as void fraction, mass fraction, pressure and velocity contours.

It is clear that the full cavitation model used in this simulation includes a plethora of effects ignored in previous studies, such as unsteady, fluid viscosity, turbulence and slip speed factor (ratio between actual and theoretical impeller rotating speed).

\section{Boundary Conditions}

In the case of a four-blade axial flow pump, sixteen blocks were used to generate the $3 \mathrm{D}$ impeller model as shown in figure 2 . The

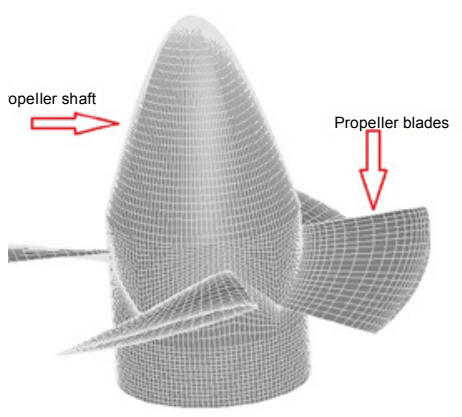

Figure 2: Geometry technique for the axial pump. 
structured grid consists of about 180,000 nodes. The propeller diameter is 4 in. $(101 \mathrm{~mm})$. Reynolds number is about 333,000 . The blade angles were $60^{\circ}, 70^{\circ}$ and $80^{\circ}$. In order to simulate the rotation in the structured gird of the rotor, a rotating frame of the grid was assumed around the rotor axis, while the slip speed factor of the rotating frame is equal to 0.67. The computer used was Intel Core i5 Processor $2.4 \mathrm{GHz}$, and 4 GB RAM.

\section{Results and Discussion}

Two comparisons between experimental visualizations and numerical results are presented in figure 3. A reliable qualitative agreement between experiments and calculation can be observed: the tip cavitating structure is correctly obtained in the two cases as shown in figure 3 . The shape of the cavitation sheet predicted numerically is particularly close to the one observed in experiments using a high speed camera of $1000 \mathrm{fps}$. The cavitation tip, which seems to be characterized experimentally by a rather low void ratio, is also qualitatively well simulated by the model. It is not significant that its precise shape is not the same in experiment and in the calculation, since this structure is highly fluctuating in time and in space.

The axial pump performance curves at blade angles $60^{\circ}, 70^{\circ}$ and $80^{\circ}$ is displayed in figures 4,5 and 6 respectively for the relations of; (1) total head ${ }^{\triangle P}$ (bar), (2) mechanical power $P_{m}$ (Watt), and (3) overall efficiency $\eta_{p}(\%)$,versus the flow rate $Q(\mathrm{~L} / \mathrm{s})$ at $3000 \mathrm{rpm}$. It is apparent that as the flow rate increases the total head decreases, the mechanical power decreases and the pump efficiency increases up to the best efficiency point. From then on, the efficiency decreases.

\section{Cavitation Simulation}

The contours of computed vapor volume fraction on the rotor at blade angles $80^{\circ}, 70^{\circ}, 60^{\circ}$ are presented in figure $7 \mathrm{a}, \mathrm{b}, \mathrm{c}$ respectively. It is
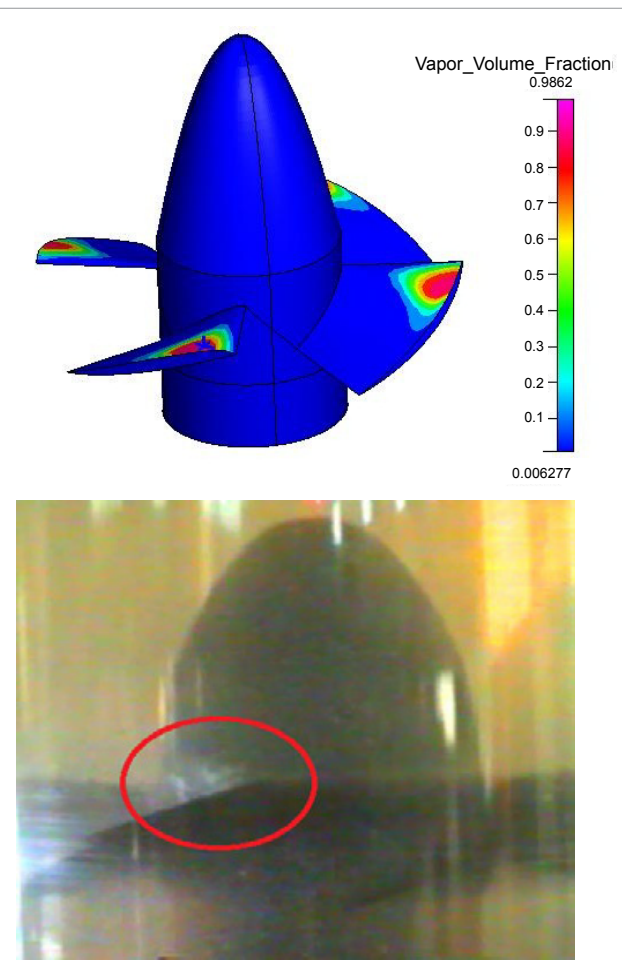

Figure 3: Cavitation at the impeller of the axial pump: Experimental and numerical results comparison.

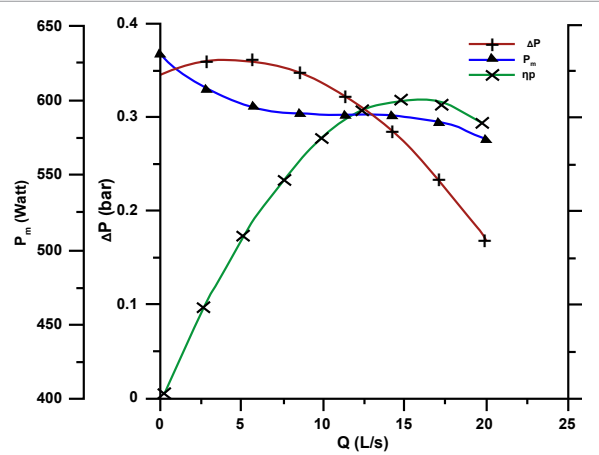

Figure 4: Experimental performance curve of axial pump, $3000 \mathrm{rpm}$, $\beta_{1}=60^{\circ}$ and $\beta_{2}=60^{\circ}$.

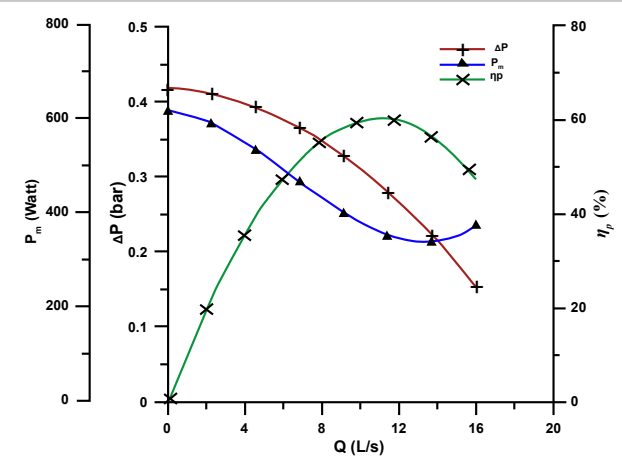

Figure 5: Experimental performance curve of axial pump, $3000 \mathrm{rpm}, \beta_{1}=70^{\circ}$ and $\beta_{2}=70^{\circ}$.

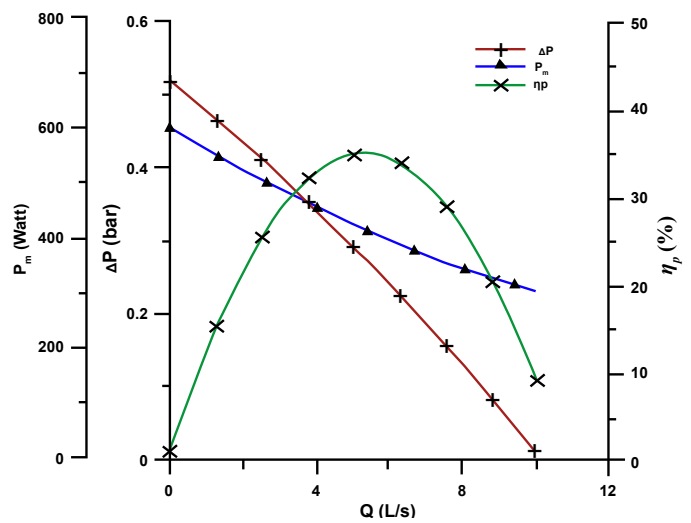

Figure 6: Experimental performance curve of axial pump, $3000 \mathrm{rpm}, \beta_{1}=80^{\circ}$ and $\beta_{2}=80^{\circ}$.

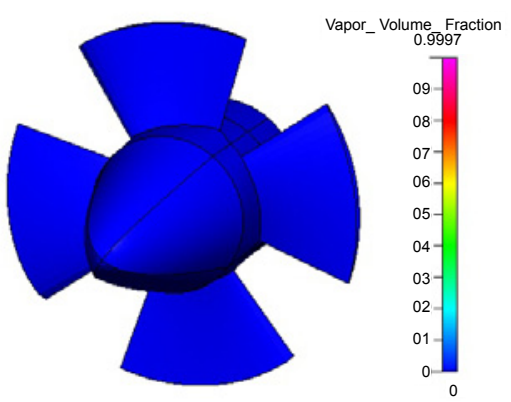

Figure 7a: Vapor volume distribution on the rotor at $3000 \mathrm{rpm}, \beta_{1}=80^{\circ}$ and $\beta_{2}=80^{\circ}$. 


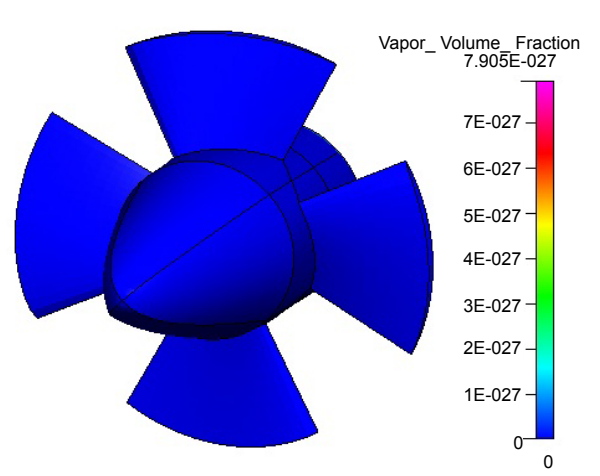

Figure 7b: Vapor volume distribution on the rotor at $3000 \mathrm{rpm}, \beta_{1}=70^{\circ}$ and $\beta_{2}=70^{\circ}$.

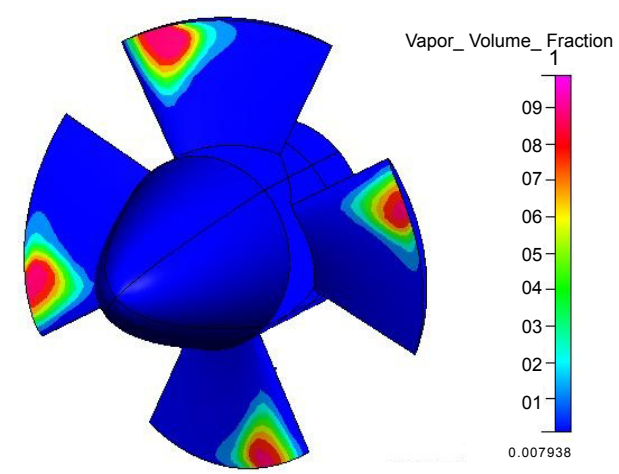

Figure 7c: Vapor volume distribution on the rotor at $3000 \mathrm{rpm}, \beta_{1}=60^{\circ}$ and $\beta_{2}=60^{\circ}$.
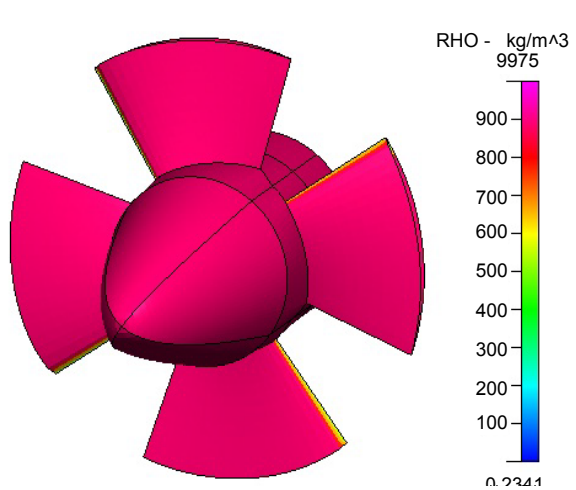

Figure 8a: Density distribution on the rotor at $3000 \mathrm{rpm}, \beta_{1}=80^{\circ}$ and $\beta_{2}=80^{\circ}$.

obvious that cavitation does not appear at blade angles $80^{\circ}$ and $70^{\circ}$ while it is present at blade angle $60^{\circ}$ at the leading edge of the suction side due to the decrease in static pressure below the vapor pressure. The computed density contours on the rotor at blade angles $80^{\circ}, 70^{\circ}, 60^{\circ}$ are presented in figure $8 \mathrm{a}, \mathrm{b}, \mathrm{c}$ respectively. It is obvious that is no density at blade angles $80^{\circ}$ and $70^{\circ}$, while the density decreases sharply at blade angles $60^{\circ}$ at the leading edge of the blades due to the decrease in static pressure below the vapor pressure.

The density distribution on the hydrofoil at the tip section at blade angles $80^{\circ}, 70^{\circ}$ and $60^{\circ}$ is shown in figure 9 a. Again, there is no density change at blade angles $70^{\circ}$ and $80^{\circ}$, while the density decreases sharply at the leading edge of the blades at blade angle $60^{\circ}$. The static pressure distribution on the hydrofoil at the tip section at blade angles $80^{\circ}, 70^{\circ}$ and $60^{\circ}$ is shown in Figure 9b. The static pressure decreases sharply below the vapor pressure at the leading edge of the blades at blade angle $60^{\circ}$, while the static pressure at blade angles $70^{\circ}$ and $80^{\circ}$ is above the vapor pressure. The vapor volume distribution on the hydrofoil at tip section at blade angles $80^{\circ}, 70^{\circ}$ and $60^{\circ}$ is shown in figure $9 \mathrm{c}$. The vapor volume fraction increases sharply at the leading edge of the blades at blade angle $60^{\circ}$, while at blade angles $70^{\circ}$ and $80^{\circ}$ is equal to zero. The total pressure distribution on the hydrofoil at tip section at blade angles $80^{\circ}, 70^{\circ}$ and $60^{\circ}$ is shown in figure $9 \mathrm{~d}$. The total pressure decreases sharply below the vapor pressure at the leading edge of the blades at blade angle $60^{\circ}$, while at blade angles $70^{\circ}$ and $80^{\circ}$ is above the vapor pressure.

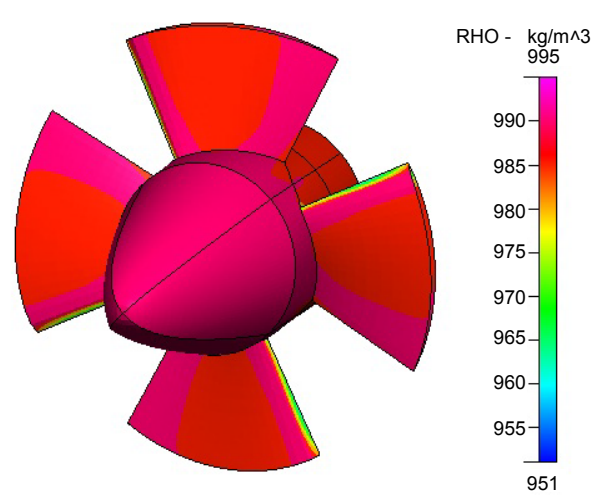

Figure 8b: Density distribution on the rotor at $3000 \mathrm{rpm}, \beta_{1}=70^{\circ}$ and $\beta_{2}=70^{\circ}$.
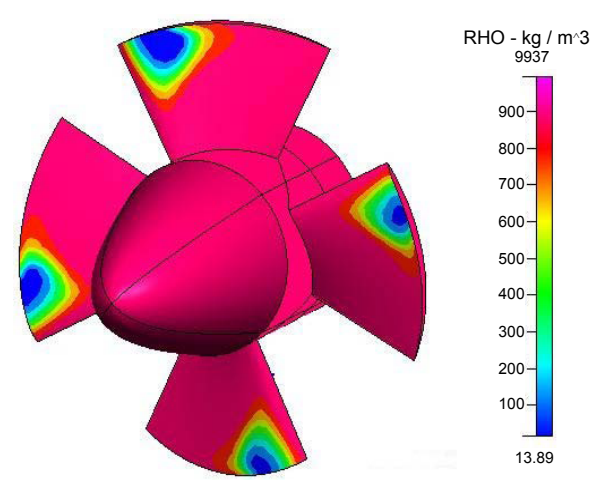

Figure 8c: Density distribution on the rotor at $3000 \mathrm{rpm}, \beta_{1}=60^{\circ}$ and $\beta_{2}=60^{\circ}$.

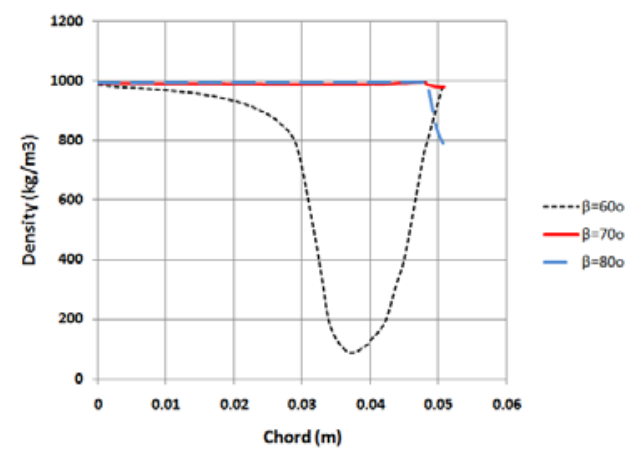

Figure 9a: Density distribution on the suction side of the hydrofoil at variable blade angles and $3000 \mathrm{rpm}$. 
Citation: Mostafa NH, Adel M (2012) Effect of Blade Angle on Cavitation Phenomenon in Axial Pump. J Appl Mech Eng 1:112. doi:10.4172/21689873.1000112

Page 5 of 6

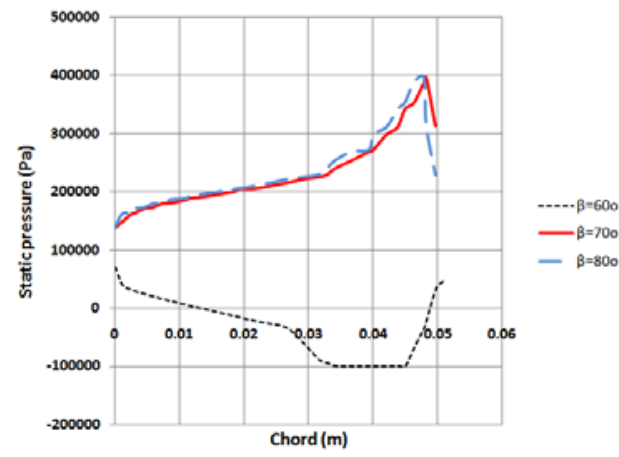

Figure 9b: Static Pressure distribution on the suction side of the hydrofoil at variable blade angles and $3000 \mathrm{rpm}$.

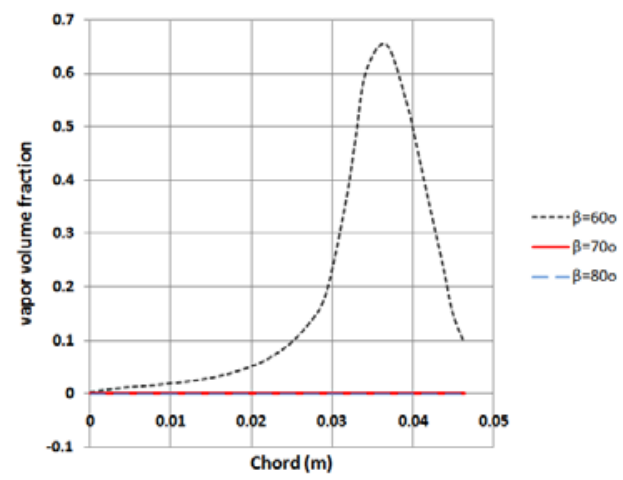

Figure 9c: Vapor volume fraction distribution on suction side of the hydrofoil at variable blade angles and $3000 \mathrm{rpm}$.

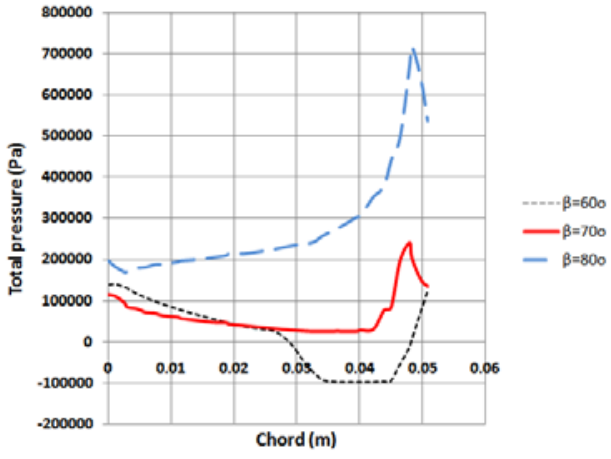

Figure 9d: Total pressure on the suction side of the hydrofoil at variable blade angles and $3000 \mathrm{rpm}$.

\section{Summary and Conclusions}

An experimental and numerical study was performed to investigate the effect of blade angle on the cavitation phenomenon in axial pump. The CFD-ACE + code $[10,11]$ has been validated through comparison of predicted numerical with experimental results. The solution of flow in the axial machine is numerically feasible. Also, the code was validated to simulate the cavitation phenomenon in axial pump.

From the results of the present study, the following conclusions are drawn:

1. There is good agreement between computational and experimental results. This implies that the code has the potential to simulate void growth and cavitation in rotating machines.

2. The comparison between the numerical and experimental results shows a good matching of bubble formation with the exemption of the computational bubble shape that has small dissimilarity. This may be attributed to the assumption of similar passages in numerical simulation.

3. The results show that tip cavitation can directly lead to losses in efficiency, especially for flow rates inside the cavitation zone.

4. In axial pump cavitation occurred at blade angle $60^{\circ}$ and not appeared at blade angles $70^{\circ}$ and $80^{\circ}$. This is an important result, since just adjusting the blade angle to $70^{\circ}$ or $80^{\circ}$, cavitation can be avoided.

5. Corresponding to the pressure distribution, the highest cavity void fraction area appears where the pressure takes its lowest value on the suction side of blade.

6. As the blade angle increases from $60^{\circ}$ to $80^{\circ}$ the total head of axial pump decreases sharply, also the flow rate ranges decreases.

\section{Nomenclature}

\begin{tabular}{|l|l|l|}
\hline \multicolumn{1}{|c|}{$C_{e}, C_{c}$} & Phase change rate coefficients & \\
\hline$C_{N}$ & $\begin{array}{l}\text { Slip speed factor "ratio between impeller actual } \\
\text { rotational speed and theoretical rotating frame speed" }\end{array}$ & \\
\hline$f$ & Vapor mass fraction & $\mathrm{m}^{2} / \mathrm{s}^{2}$ \\
\hline $\mathrm{K}$ & Turbulence kinetic energy & $\mathrm{rpm}$ \\
\hline $\mathrm{N}$ & Pump rotational speed & $\mathrm{N} / \mathrm{m}^{2}$ \\
\hline $\mathrm{P}$ & Fluid static pressure & $\mathrm{N} / \mathrm{m}^{2}$ \\
\hline$P_{\text {sat }}$ & Saturation pressure & $\mathrm{N} / \mathrm{m}^{2}$ \\
\hline$P_{\text {turb }}^{\prime}$ & The magnitude of pressure fluctuations & $\mathrm{N} / \mathrm{m}^{2}$ \\
\hline$P_{t}$ & Total pressure & $\mathrm{N} / \mathrm{m}^{2}$ \\
\hline$P_{V}$ & Vapor pressure & $\mathrm{Nm} / \mathrm{Kg} \cdot \mathrm{k}$ \\
\hline$R$ & Universal gas constant & \\
\hline$R$ & Phase change rate & \\
\hline$R e$ & Reynolds number & $\mathrm{K}$ \\
\hline$T$ & Fluid temperature & $\mathrm{S}$ \\
\hline$\Delta t$ & Physical time step & $\mathrm{Kg} / \mathrm{Kg}-\mathrm{mol}$ \\
\hline W & Molecular weight & \\
\hline
\end{tabular}

\section{Greek Letters}

\begin{tabular}{|l|l|c|}
\hline$\beta_{1}$ & $\begin{array}{l}\text { Blade inlet angle (angle between the tangent to camber } \\
\text { line at inlet and the axial direction) }\end{array}$ & degree \\
\hline$\beta_{2}$ & $\begin{array}{l}\text { Blade outlet angle (angle between the tangent to camber } \\
\text { line at outlet and the axial direction) }\end{array}$ & degree \\
\hline$\alpha_{v}$ & Vapor volume fraction & \\
\hline$\alpha_{g}$ & gas volume fraction & -- \\
\hline$\sigma$ & Cavitation number $\left(\left(p_{\infty}-p_{v}\right) /\left(1 / 2 \rho, u^{2}\right)\right)$ & $\mathrm{Kg} / \mathrm{m}^{3}$ \\
\hline$\rho$ & The mixture density & \\
\hline
\end{tabular}

\section{Suffixes}

\begin{tabular}{|l|l|}
\hline C & Bubble reduction and collapse \\
\hline e & Bubble generation and expansion \\
\hline Gas, G & Gaseous phase \\
\hline L & Liquid phase \\
\hline V & Vapor phases \\
\hline
\end{tabular}


Citation: Mostafa NH, Adel M (2012) Effect of Blade Angle on Cavitation Phenomenon in Axial Pump. J Appl Mech Eng 1:112. doi:10.4172/21689873.1000112

\section{References}

1. Sudsuansee T, Nontakaew U, Tiaple $Y$ (2011) Simulation of leading edge cavitation on bulb turbine. Songklanakarin J Sci Technol 33: 51-60.

2. Mostafa NH, Rayan MA, Mahgob MM (1990) Energetic model for cavitation erosion prediction in centrifugal pump impeller. Proceeding of ASME/CSME Cavitation and Multiphase flow forum, Toronto, Canada.

3. Fukaya M, Okamura T, Tamura Y, Matsumoto Y (2003) Prediction of Cavitation Performance of Axial Flow Pump by Using Numerical Cavitating Flow Simulation with Bubble Flow Model. Fifth International Symposium on Cavitation, Osaka, Japan

4. Sedlář M, Zima P, Němec T, Maršík F (2008) Analysis of Cavitation Phenomena in Water and its Application to Prediction of Cavitation Erosion in Hydraulic Machinery. ICPWS XV, Berlin.

5. De-sheng Z, Wei-dong S, Bin C, Xing-fan G (2010) Unsteady flow analysis and experimental investigation of axial-flow pump. Journal of Hydrodynamics, Ser. B 22: 35-43

6. Tabar MTS, Poursharifi Z (2011) An Experimental Study of Tip Vortex Cavitation Inception in an Axial Flow Pump. World Academy of Science, Engineering and Technology 73: 527-530.

7. Hinze JO (1975) Turbulence. ( $2^{\text {nd }}$ Edn) McGraw-Hill Book Company, New York, USA.

8. Hammitt FG (1980) Cavitation and multiphase flow phenomena. McGraw-Hil Book Company, New York, USA.

9. Pereira F, Salvatrore F, Felice F (2004) Measurement and Modeling of propeller cavitation in uniform inflow. J Fluid Eng- TASME 126: 671-679.

10. CFD-ACE+ V2008 (2008) User Manual - Volume 1.

11. CFD-ACE+ V2008 (2008) Modules Manual - Volume 1. 